\title{
Barriers and Facilitators of Implementing WHO Safe Surgery Checklist: A Cross-Sectional Study in Public Hospitals of Iran
}

\author{
Milad Khodavandi ${ }^{1}$, Edris Kakemam ${ }^{2}$, Shabnam Ghasemyani (iD ${ }^{3}$ and Rahim Khodayari-Zarnaq (iD ${ }^{4,}$ \\ ${ }^{1}$ Student Research Committee, School of Management and Medical Informatics, Tabriz University of Medical Sciences, Tabriz, Iran \\ ${ }^{2}$ School of Management and Medical Informatics, Tabriz University of Medical Sciences, Tabriz, Iran \\ ${ }^{3}$ Department of Health Services Management, School of Health Management and Information Sciences, Iran University of Medical Sciences, Tehran, Iran \\ ${ }^{4}$ Department of Health Policy and Management, School of Management and Medical Informatics, Tabriz University of Medical Sciences, Tabriz, Iran \\ Corresponding author: Tabriz Health Services Management Research Center, Tabriz University of Medical Sciences, Tabriz, Iran. Email: rahimzarnagh@gmail.com
}

Received 2021 July 22; Revised 2021 October 23; Accepted 2021 November 08.

\begin{abstract}
Background: The implementation of WHO safe surgery checklist (SSC) was proposed by the Ministry of Health and Medical Education in the operation rooms of Iranian hospitals in 2011, but was canceled after several years due to some challenges in implementation.

Objectives: This study aimed to investigate the barriers and facilitators of the effective implementation of the SSC.

Methods: This cross-sectional study was performed in public hospitals of Tabriz city in 2019. The study population consisted of operation room working staff, and the purposive sampling was used. The research tool was a researcher-made questionnaire designed through literature review and included three parts of demographic variables, barriers, and facilitating factors. Data were analyzed with SPSS-22.

Results: The mean of barriers to implementation of the SSC was 3.03 out of 5. The most important barrier to implementing the checklist was the weakness in team working (3.18), while checklist barriers were the least important (2.98). The mean score of facilitating factors was 3.46. Among the facilitators, the highest score was for team working facilitators with a score of 3.47 and the lowest score was for the facilitators associated with the checklist with a score of 3.37.

Conclusions: This is one of the first studies that explored the barriers and facilitators of SSC implementation in Iranian hospitals. We identified weak team working as the most important barrier to implementing the checklist. These results help policymakers and hospital managers to implement the checklist more effectively.
\end{abstract}

Keywords: Facilitators, Safe Surgical Checklist, Barriers

\section{Background}

Annually, 234 million patients undergo surgery procedures, adverse events in surgery were reported to occur in more than $14 \%$ of patients (1), and $55 \%$ of these adverse events are preventable (2). The safe surgery checklist (SSC) was introduced by WHO in 2008 to be used in operation rooms (3). The SSC was developed to improve teamwork, communication, consistency of care (4), prevent adverse events, strengthen safety practices, and improve the quality of care provided to the surgical patient (5). A study conducted in Norwegian hospitals found that safe surgery checklist have reduced mortality rate (4).

The order for SSC implementation of WHO has been issued by the MOH in most of the country's operation rooms since 2011 (6). The SSC has been demonstrated to reduce surgical complications and deaths by 30 - 50\% (7). However, the order was canceled due to the challenges in the application of a safety checklist, and now its application is not mandatory. Several studies have been conducted to evaluate the effects of applying SSCs, and their results have shown improved safety status $(8,9)$.

\section{Objectives}

The present research was conducted to determine the barriers and facilitators of effective implementation of SSCs in public hospitals of Tabriz city.

\section{Methods}

This study was a descriptive cross-sectional investigation performed in nine public hospitals in Tabriz in 2019. The study population consisted of operation room staff, including surgeons, anesthesiologists, and nurses. The total population employed was 220 participants. The purposive 
sampling method was used to select the samples. The participants were familiar with the operating room checklist.

In this study, a researcher-made questionnaire was used according to the previous studies (10-14). The questionnaire includes three parts: demographic variables, barriers, and factors that facilitate SSC efficient implementation. Barriers to effective implementation of the SSC were divided into four dimensions, including organizational barriers, systemic barriers, team barriers, and checklist-related barriers. The facilitating factors also have four organizational, systemic, team, and checklist-related dimensions. The validity of the questionnaire was confirmed by health management specialists, surgeons, and anesthesiologists. Its reliability was also confirmed by Cronbach's alpha coefficient to be over 70\% for all dimensions. Required explanations were given to the participants about the purpose of the research. Participation in the study was completely voluntary, and verbal consent was obtained from all participants. Data were analyzed by SPSS 22 software. Descriptive statistics such as mean \pm $\mathrm{SD}$, percentage, and frequency were used to describe the variables. To determine data normality, the KolmogorovSmirnov test was used. The differences between the mean scores of barriers and facilitators among the three groups of surgeons, anesthesiologists, and nurses and also were assessed by ANOVA test.

\section{Results}

Twelve surgeons, 12 anesthesiologists, and 115 nurses participated in the study. The response rate was $63 \%$. The mean \pm SD of participants' age was $34.29 \pm 8.52$. The majority of participants were female (65.5\%). The mean \pm SD of clinical experience of the respondents was $9.72 \pm 7.6$ years. Of the total 139 participants, only 16 had managing experience in the operating room. The majority of participants had a Bachelor's degree (71\%) (Table 1).

The mean \pm SD of barriers to effective implementation of the SSC in all dimensions was $3.03 \pm 0.55$ with a minimum score of 1.37 and a maximum score of 4.79 (

The mean score of facilitators for the effective implementation of the SSC in all dimensions was $3.46 \pm 0.76$ with a minimum score of 1.75 and a maximum score of 5 out of 5 (Table 3 ).

\section{Discussion}

The most effective barrier to the effective implementation of checklist was the dimension of team barriers. Poor communications, as well as the unclear role of each team member in completing the checklist, created these difficulties. In a study conducted by Fourcade et al., poor communication between the surgical team was one of the most important barriers (11). Also, Waehle et al.'s study showed that the most important challenge proposed by nurses was acceptance and commitment to implement the checklist (14).

The lack of a culture of change and the way the checklists were introduced and implemented were reported as the organizational barriers that come to the surface at the initiation of the checklist implementation, while failing to resolve them may pave the way for future barriers. The dimension of the systemic barriers gained the third score. Time-consuming completion of checklists, which often have a large workload, was considered an obstacle. In a study conducted by Kariyoi et al., insufficient time to use a checklist and not having a priority to use the checklist were identified as barriers (12).

According to the results, the barriers associated with the checklist itself were less important than other barriers. It can be argued that the localization of the checklist was successful. However, the three steps of completing the checklist caused the mental involvement of team members, wasting time, and creating conflict. In a study by Fourcade et al., checklist-related barriers were reported to be the most important barrier (11). This was in apposite to the findings of this study.

The overall results indicated the importance of facilitators in all dimensions. Team facilitators with the slightest difference achieved the highest score among the other dimensions. Involving operation room staff in completing checklist and actively leading the team by the senior members can facilitate the implementation of the checklist. According to Verwey et al.'s study, implementing checklist required the support of all operation room staff (15). In the study by Tostes et al., the need for an effective leader in the operation room is significant to complete the checklist and motive team members in its completion (5).

The organizational dimension of facilitators was the second most important dimension. Granting legal responsibility to the surgical team was an important facilitator to respond to any failure in completing the checklist. Organization managers' support and staff training for proper checklist implementation are important facilitators of this dimension. In an investigation by Sokhanvar et al., the support of the managers was considered an important factor in facilitating the checklist implementation (6). According to Mahajan et al.'s study, organizational support, organizational leadership, and training were required for successful implementation (13).

Implementation of checklists in operation rooms requires the identification and removal of barriers for its effective implementation, as well as designing a process and the method of its implementation in a transparent manner. In addition to removing barriers, to facilitate the implementation of checklists, we must pay attention to the working procedures such as training and introducing the process, support of the managers, permanent audit of the 
Khodavandi M et al.

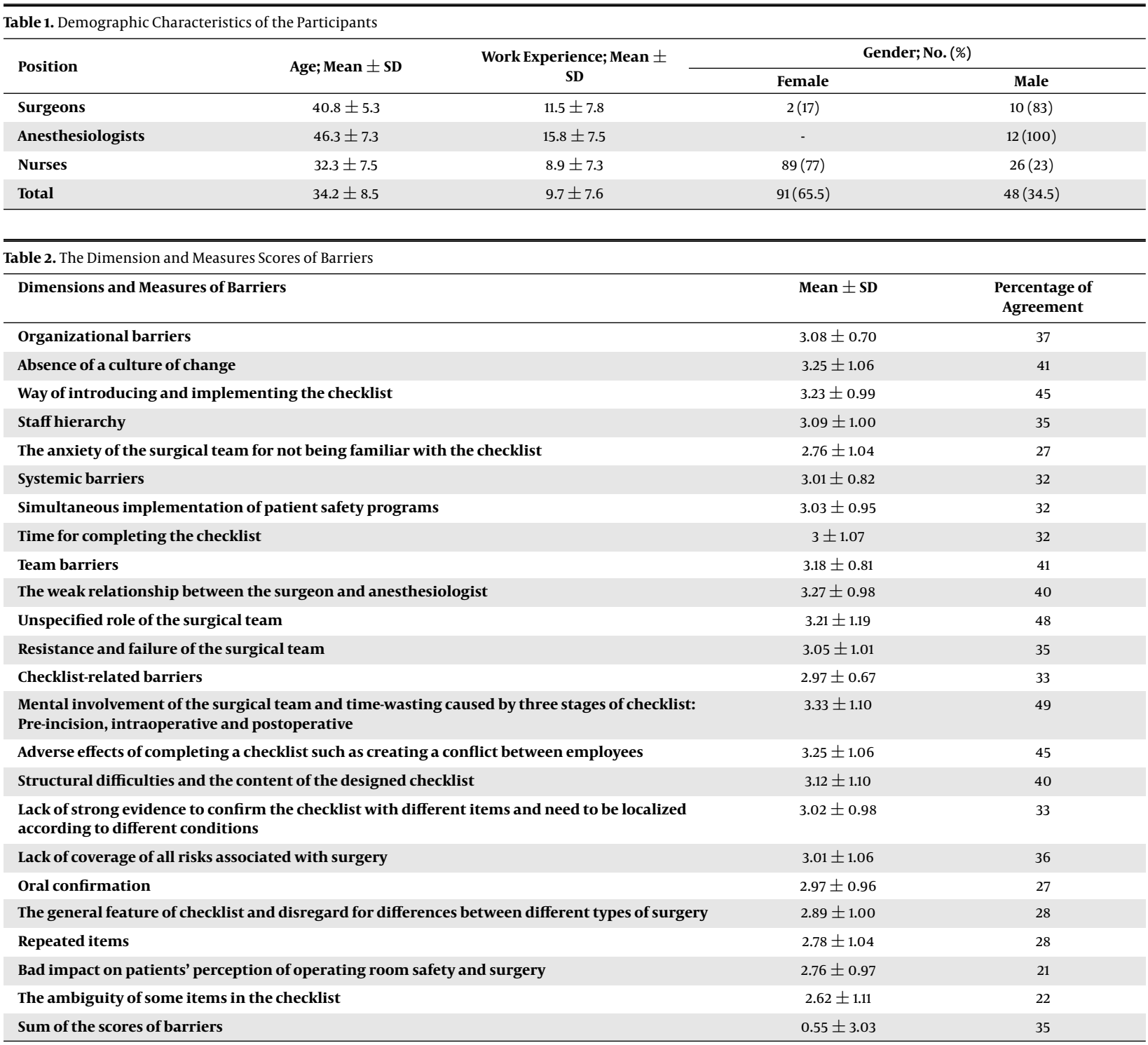

checklist completion process, integrating checklist implementation with other patient safety promoting programs, and most importantly, the legal responsibility of the staff to respond to the failure of checklist implementation.

\section{Footnotes}

Authors' Contribution: Khodayari-Zarnaq R and Khodavandi $\mathrm{M}$, designed the research; Khodavandi $\mathrm{M}$ and Kakemam E, conducted research; Khodayari-zarnaq R, Khodavandi $M$, and Ghasemyani S, analyzed data; KhodayariZarnaq R, Ghasemyani S, and Kakemam E, wrote the manuscript. Khodayari-zarnaq $\mathrm{R}$ had primary responsibility for the final content. All authors read and approved the final manuscript.
Conflict of Interests: The authors declare that they have no competing interests.

Ethical Approval: The protocol of this study was approved by the Central Ethics Committee of Tabriz University of Medical Sciences (TUOMS) under the code IR.TBZMED.REC.2018.153.

Funding/Support: This study was funded by the Tabriz Health Services Management Research Center.

Informed Consent: Participation in the study was completely voluntary, and verbal consent was obtained from all participants. Participants were informed of the objectives of the study. All questionnaires were anonymous, and the data were kept confidential. 


\begin{tabular}{|c|c|c|}
\hline Dimensions and measures of facilitators & Mean & $\begin{array}{c}\text { Percentage of } \\
\text { agreement }\end{array}$ \\
\hline Organizational facilitators & $3.45 \pm 0.55$ & 49 \\
\hline $\begin{array}{l}\text { The surgical team is legally responsible for responding to the checklist in the case that it is not } \\
\text { completed }\end{array}$ & $3.65 \pm 0.87$ & 63 \\
\hline Supporting managers of the organization & $3.54 \pm 0.96$ & 58 \\
\hline $\begin{array}{l}\text { Complete training, and introduction of the process and related checklist prior to its } \\
\text { implementation }\end{array}$ & $3.48 \pm 0.89$ & 48 \\
\hline Permanent audit of the completion process & $3.33 \pm 0.80$ & 42 \\
\hline Lack of focusing on the organizational hierarchy & $3.20 \pm 0.91$ & 33 \\
\hline Systemic facilitator & $3 / 41 \pm 0.93$ & 48 \\
\hline Team facilitators dimensions & $3.46 \pm 0.76$ & 50 \\
\hline Participation of surgical team in completing and modifying & $3.59 \pm 0.95$ & 55 \\
\hline Institutionalizing the checklist by supporting senior members of the surgical team & $3.45 \pm 0.96$ & 53 \\
\hline Improving leadership skills & $3.43 \pm 0.94$ & 47 \\
\hline Active and effective leadership & $3.37 \pm 0.92$ & 47 \\
\hline Checklist-related facilitator & $3.37 \pm 1.07$ & 42 \\
\hline Reviewing the checklist based on the feedback of the surgical team & $3.37 \pm 1.07$ & 42 \\
\hline Sum of the scores of barriers & $0.76 \pm 3.46$ & 49 \\
\hline
\end{tabular}

\section{References}

1. Gillespie BM, Harbeck EL, Lavin J, Hamilton K, Gardiner T, Withers TK, et al. Evaluation of a patient safety programme on Surgical Safety Checklist Compliance: A prospective longitudinal study. BMJ Open Qual. 2018;7(3). e000362. doi: 10.1136/bmjoq-2018-000362. [PubMed: 30057963]. [PubMed Central: PMC6059267].

2. Haugen AS, Sevdalis N, Softeland E. Impact of the World Health Organization Surgical Safety Checklist on Patient Safety. Anesthesiology. 2019;131(2):420-5. doi: 10.1097/ALN.0000000000002674. [PubMed: 31090552].

3. Willassen ET, Jacobsen ILS, Tveiten S. Safe Surgery Checklist, Patient Safety, Teamwork, and Responsibility-Coequal Demands? A Focus Group Study. Glob Qual Nurs Res. 2018;5:2333393618764070. doi: 10.1177/2333393618764070. [PubMed: 29623287]. [PubMed Central: PMC5881961].

4. Haugen AS, Waehle HV, Almeland SK, Harthug S, Sevdalis N, Eide GE, et al. Causal Analysis of World Health Organization's Surgical Safety Checklist Implementation Quality and Impact on Care Processes and Patient Outcomes: Secondary Analysis From a Large Stepped Wedge Cluster Randomized Controlled Trial in Norway. Ann Surg. 2019;269(2):283-90. doi: 10.1097/SLA.0000000000002584. [PubMed: 29112512]. [PubMed Central: PMC6326038].

5. Tostes M, Galvao CM. Surgical safety checklist: benefits, facilitators, and barriers in the nurses' perspective. Rev Gaucha Enferm. 2019;40(spe). e20180180. doi: 10.1590/1983-1447.2019.20180180. [PubMed: 30652803].

6. Sokhanvar M, Kakemam E, Goodarzi N. Implementation of the surgical safety checklist in hospitals of Iran; operating room personnel's attitude, awareness and acceptance. Int J Health Care Qual Assur. 2018;31(6):609-18. doi: 10.1108/IJHCQA-03-2017-0051. [PubMed: 29954264].

7. Hellar A, Tibyehabwa L, Ernest E, Varallo J, Betram MM, Fitzgerald L, et al. A Team-Based Approach to Introduce and Sustain the
Use of the WHO Surgical Safety Checklist in Tanzania. World $J$ Surg. 2020;44(3):689-95. doi: 10.1007/s00268-019-05292-5. [PubMed: 31741072].

8. Askarian M, Kouchak F, Palenik CJ. Effect of surgical safety checklists on postoperative morbidity and mortality rates, Shiraz, Faghihy Hospital, a 1-year study. Qual Manag Health Care. 2011;20(4):293-7. doi: 10.1097/QMH.ob013e318231357c. [PubMed: 21971026].

9. Mohebbifar R, Purrostami K, Mahdavi A, Hassanpoor E, Sokhanvar M, Nazari M, et al. [Effect of Surgical Safety Checklist on Mortality of Surgical Patients in the $\alpha$ University Hospitals]. Alborz University Medical Journal. 2014;3(1):33-9. Persian. doi: 10.18869/acadpub.aums.3.1.33.

10. Adib Haj-Bagheri M, Parvizi S, Salsali M.[Qualitative Research Methods]. Boshra: Tehran, Iran; 2013. Persian.

11. Fourcade A, Blache JL, Grenier C, Bourgain JL, Minvielle E. Barriers to staff adoption of a surgical safety checklist. BMJ Qual Saf. 2012;21(3):191-7. doi: 10.1136/bmjqs-2011-000094. [PubMed: 22069112]. [PubMed Central: PMC3285141].

12. Kariyoi PC, Hightoweri J, Ndihokubwayoii JB, Tumusiimei P, Mwikisai C. Challenges facing the introduction of the WHO surgical safety checklist: a short experience in African countries. African Health Monitor. 2013;16(9):36-9.

13. Mahajan RP. The WHO surgical checklist. Best Pract Res Clin Anaesthesiol. 2011;25(2):161-8. doi: 10.1016/j.bpa.2011.02.002. [PubMed: 21550541].

14. Waehle HV, Haugen AS, Softeland E, Hjalmhult E. Adjusting team involvement: a grounded theory study of challenges in utilizing a surgical safety checklist as experienced by nurses in the operating room. BMC Nurs. 2012;11:16. doi: 10.1186/1472-6955-11-16. [PubMed: 22958326]. [PubMed Central: PMC3499446].

15. Verwey S, Gopalan PD. An investigation of barriers to the use of the World Health Organization Surgical Safety Checklist in theatres. S Afr Med J. 2018;108(4):336-41. doi: 10.7196/SAMJ.2017.v108i4.12780. [PubMed: 29629686]. 